\title{
Minimizing the condition number of a positive definite matrix by completion
}

\author{
L. Elsner ${ }^{1, \star}$, C. $\mathbf{H e}^{2, \star \star}$, V. Mehrmann ${ }^{2, * *}$ \\ ${ }^{1}$ Fakultät für Mathematik, Universität Bielefeld, D-33615 Bielefeld, Germany \\ 2 Fachbereich Mathematik, PSF 964, TU-Chemnitz-Zwickau, D-09009 Chemnitz, Germany \\ Received October 15, 1993
}

Summary. We consider the problem of minimizing the spectral condition number of a positive definite matrix by completion:

$$
\min \left\{\operatorname{cond}\left(\left[\begin{array}{cc}
A & B^{\mathrm{H}} \\
B & X
\end{array}\right]\right):\left[\begin{array}{cc}
A & B^{\mathrm{H}} \\
B & X
\end{array}\right] \text { positive definite }\right\},
$$

where $A$ is an $n \times n$ Hermitian positive definite matrix, $B$ a $p \times n$ matrix and $X$ is a free $p \times p$ Hermitian matrix. We reduce this problem to an optimization problem for a convex function in one variable. Using the minimal solution of this problem we characterize the complete set of matrices that give the minimum condition number.

Mathematics Subject Classification (1991): 65F35, 15A12

\section{Introduction}

Let $A$ be an $n \times n$ positive definite Hermitian matrix (denoted by $A>0$ ), let be $B$ a $p \times n$ matrix and

$$
W(X)=\left[\begin{array}{cc}
A & B^{\mathrm{H}} \\
B & X
\end{array}\right]
$$

for a Hermitian $X$. (Here $B^{\mathrm{H}}$ denotes the conjugate transpose of the matrix B.)

We consider the optimization problem

$$
\min _{X, W(X)>0} \operatorname{cond}(W(X)),
$$

where

$$
\operatorname{cond}(W(X))=\|W(X)\|\left\|W(X)^{-1}\right\|=\frac{\lambda_{\max }(W(X))}{\lambda_{\min }(W(X))}
$$

\footnotetext{
* Research supported by SFB 343 Diskrete Strukturen in der Mathematik, Universität Bielefeld

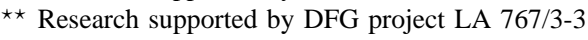

Correspondence to: L. Elsner
} 
is the spectral condition number [6]. (Here $\lambda_{\max }(W), \lambda_{\min }(W)$ denote the maximal and minimal eigenvalue of the matrix $W$ and \|\| denotes the spectral norm.)

This problem arises in the construction of optimal feedback control for descriptor systems [4] as well as in the construction of optimal preconditioners for the iterative solution of linear systems on parallel computers via divide and conquer techniques [7].

We will show that the optimization problem (1) is equivalent to a minimization problem for a convex function in one variable in combination with a matrix inequality.

Using the solution of this convex minimization problem, we can then characterize the complete set of solutions to (1).

The idea of treating such kind of optimization problems as a matrix inequality problem is not new. Davis, Kahan and Weinberger [2] used it to solve the problem of minimizing the norm of a matrix by completion and the authors applied it to the problem of minimizing the norm of the inverse of a matrix by completion [4]. See also $[1,8,5]$.

\section{Main theorem}

To formulate our result, we introduce the following functions involving the parameters $A, B$ of our problem. Define the real valued function

$$
f(t):=\left\|t^{2} B^{\mathrm{H}} B+(I-t A)^{2}\right\|
$$

and for $\tau$ not in the spectrum of $A$ the matrix valued function

$$
X(\tau):=\tau I+B(A-\tau I)^{-1} B^{\mathrm{H}} .
$$

It is easy to see that $f(t)$ is strictly convex, as it is the maximum over all $x \neq 0$ of the strictly convex function

$$
\frac{x^{\mathrm{H}}\left(t^{2} B^{\mathrm{H}} B+(I-t A)^{2}\right) x}{x^{\mathrm{H}} x} .
$$

Since

$$
\frac{d}{d \tau} X(\tau)=I+B(A-\tau I)^{-2} B^{\mathrm{H}}>0,
$$

it follows that $X(\tau)$ is monotone in any interval not containing an eigenvalue of $A$.

Our main result then is as follows:

Theorem 1. Let $A$ be an $n \times n$ positive definite Hermitian matrix and let $B$ be $a p \times n$ complex matrix. Consider the completion problem of finding an Hermitian positive definite matrix $X^{*}$ such that the condition number of $W\left(X^{*}\right)$, where

$$
W(X)=\left[\begin{array}{cc}
A & B^{\mathrm{H}} \\
B & X
\end{array}\right],
$$

is minimal among all $X$ such that $W(X)>0$. Then

$$
\min _{X, W(X)>0} \operatorname{cond}(W(X))=\frac{1+\sqrt{ } f^{*}}{1-\sqrt{ } f^{*}},
$$

where 


$$
f^{*}:=\min _{t \in \mathrm{R}} f(t)
$$

with $f(t)$ defined in (3).

The set of all matrices $X$ satisfying (5) is

$$
\left\{X=X^{\mathrm{H}} \in \mathbb{C}^{p, p}: \lim _{\substack{\alpha \rightarrow \alpha^{*} \\ \alpha<\alpha^{*}}} X(\alpha) \leq X \leq \lim _{\substack{\beta \rightarrow \beta^{*} \\ \beta^{*}<\beta}} X(\beta)\right\},
$$

where

$$
\alpha^{*}=\frac{1-\sqrt{ } f^{*}}{t^{*}}, \beta^{*}=\frac{1+\sqrt{ } f^{*}}{t^{*}} .
$$

and $t^{*}$ is the unique argument satisfying $f\left(t^{*}\right)=f^{*}$.

The limits in (7) both exists, even if $\alpha^{*}$ is the smallest eigenvalue of $A$ or $\beta^{*}$ is the largest eigenvalue of $A$.. All matrices $X$ in (7) have the property that $\alpha^{*}, \beta^{*}$ are the minimal, and maximal eigenvalue of $W(X)$, respectively.

Proof. We start the proof by establishing two facts:

Fact 1: For given $\alpha, \beta, \alpha<\beta$ the following two statements are equivalent:

$$
\begin{gathered}
X=X^{\mathrm{H}} \text { satisfies } \alpha I<W(X)<\beta I \\
\alpha<\lambda_{\min }(A), \lambda_{\max }(A)<\beta \text {, and } X(\alpha)<X<X(\beta) .
\end{gathered}
$$

This follows by taking Schur complements in both $W(X)-\alpha I$ and $\beta I-W(X)$.

Fact 2: For given $\alpha, \beta, \alpha<\beta$ the following four statements are equivalent:

$$
\begin{gathered}
X(\alpha)<X(\beta) \text { and } \alpha<\lambda_{\min }(A), \lambda_{\max }(A)<\beta . \\
B(A-\alpha I)^{-1}(\beta I-A)^{-1} B^{\mathrm{H}}<I, \alpha<\lambda_{\min }(A), \lambda_{\max }(A)<\beta . \\
B^{\mathrm{H}} B<(A-\alpha I)(\beta I-A) . \\
\left(\frac{\beta-\alpha}{\beta+\alpha}\right)^{2}>f\left(\frac{2}{\beta+\alpha}\right) .
\end{gathered}
$$

Here the equivalence of (11) and (12) follows from the relation

$$
\frac{1}{\beta-\alpha}(X(\beta)-X(\alpha))=I-B(A-\alpha I)^{-1}(\beta I-A)^{-1} B^{\mathrm{H}} .
$$

The equivalence of (12) and (13) is evident, since $(A-\alpha I)(\beta I-A)>0$.

Using the relation

$$
(A-\alpha I)(\beta I-A)=\left(\frac{\beta-\alpha}{2}\right)^{2} I-\left(A-\frac{\beta+\alpha}{2} I\right)^{2},
$$

we obtain the equivalence of (13) and (14), since both inequalities are equivalent to

$$
\left(\frac{\beta-\alpha}{\beta+\alpha}\right)^{2} I>\left(\frac{2}{\beta+\alpha}\right)^{2} B^{\mathrm{H}} B+\left(I-\frac{2}{\beta+\alpha} A\right)^{2} .
$$


In order to prove our result we now show first that for any $X$ with $W(X)>0$ we have

$$
\left(\begin{array}{c}
\operatorname{cond}(W(X))-1 \\
\operatorname{cond}(W(X))+1
\end{array}\right)^{2} \geq f^{*}
$$

which is the same as the inequality

$$
\operatorname{cond}(W(X)) \geq \frac{1+\sqrt{ } f^{*}}{1-\sqrt{ } f^{*}} .
$$

Let $\alpha, \beta$ be the smallest and largest eigenvalue of $W(X)$, respectively. Then for any $\epsilon>0$ we have

$$
(\alpha-\epsilon) I<W(X)<(\beta+\epsilon) I .
$$

By Facts 1 and 2 we have that (10) and hence (14) is satisfied for the pair of parameters $(\alpha-\epsilon, \beta+\epsilon)$. Therefore (14) also holds, i.e.

$$
\left(\begin{array}{c}
\beta-\alpha+2 \epsilon \\
\beta+\alpha
\end{array}\right)^{2}>f\left(\begin{array}{c}
2 \\
\beta+\alpha
\end{array}\right) \geq f^{*} .
$$

As cond $(W(X))=\frac{\beta}{\alpha}$, we obtain (17) from (20).

To establish (5) we show that we have equality in (17) if and only if

$$
\left.\lim _{\substack{\alpha \rightarrow \alpha^{*} \\ \alpha<\alpha^{*}}} X(\alpha) \leq X \leq \lim _{\substack{\beta \rightarrow \beta^{*} \\ \beta^{*}<\beta}} X(\beta)\right\},
$$

where both limits exist.

Observe that $\alpha^{*}, \beta^{*}$ as in (8) satisfy

$$
\left(\begin{array}{c}
\beta^{*}-\alpha^{*} \\
\beta^{*}+\alpha^{*}
\end{array}\right)^{2}=f\left(\begin{array}{c}
2 \\
\beta^{*}+\alpha^{*}
\end{array}\right)=f^{*}
$$

Hence for $\epsilon>0$ the pair $(\alpha, \beta)=\left(\alpha^{*}-\epsilon, \beta^{*}+\epsilon\right)$ satisfies (14) and by Fact 2 also (11), i.e.,

$$
X\left(\alpha^{*}-\epsilon\right)<X\left(\beta^{*}+\epsilon\right) \text { and } \alpha^{*} \leq \lambda_{\min }(A), \lambda_{\max }(A) \leq \beta^{*} .
$$

As $X\left(\alpha^{*}-\epsilon\right)$ is monotonically increasing in $\epsilon$ and by (23) also bounded, the left limit in (21) exists and an analogous argument yields the existence of the right limit.

Now, let $X$ be a matrix satisfying (21). Then we have

$$
X\left(\alpha^{*}-\epsilon\right)<X<X\left(\beta^{*}+\epsilon\right)
$$

by the strict monotonicity of $X(\tau)$.

By Fact 1 we then have

$$
\operatorname{cond}(W(X))<\frac{\beta^{*}+\epsilon}{\alpha^{*}-\epsilon}
$$

and hence

$$
\frac{\operatorname{cond}(W(X))-1}{\operatorname{cond}(W(X))+1}<\frac{\beta^{*}-\alpha^{*}+2 \epsilon}{\beta^{*}+\alpha^{*}}=\sqrt{ } f^{*}+\frac{2 \epsilon}{\alpha^{*}+\beta^{*}}
$$

for all $\epsilon>0$. This implies equality in (18). 
To show the converse let $X$ be any matrix satisfying (18) with equality, and let $\bar{\alpha}, \bar{\beta}$ be the minimal and maximal eigenvalues of $W(X)$, respectively. Then

$$
\overline{\bar{\beta}}=\operatorname{cond}(W(X))=\frac{1+\sqrt{ } f^{*}}{1-\sqrt{ } f^{*}}=\frac{\beta^{*}}{\alpha^{*}} .
$$

Let $\epsilon>0$. Then $(\bar{\alpha}-\epsilon) I<W(X)<(\bar{\beta}+\epsilon) I$. By Facts 1 and 2 we infer (14) for the pair $(\alpha, \beta)=(\bar{\alpha}-\epsilon, \bar{\beta}+\epsilon)$, i.e.,

$$
\left(\begin{array}{c}
\bar{\beta}-\bar{\alpha}+2 \epsilon \\
\bar{\beta}+\bar{\alpha}
\end{array}\right)^{2}>f\left(\begin{array}{c}
2 \\
\bar{\beta}+\bar{\alpha}
\end{array}\right)
$$

and for $\epsilon \rightarrow 0$ we have

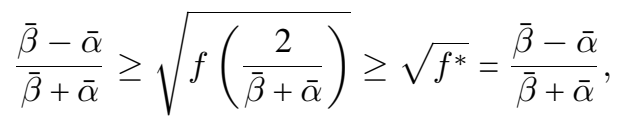

where the last equality follows from (26).

Since $f$ is strictly convex, it follows that

$$
\frac{2}{\bar{\beta}+\bar{\alpha}}=t^{*}=\frac{2}{\alpha^{*}+\beta^{*}} .
$$

Together with (26) it follows that $\bar{\alpha}=\alpha^{*}$ and $\bar{\beta}=\beta^{*}$ and by (24) we obtain (21). This finishes the proof.

To actually calculate $t^{*}$ we remark that $t^{*} \in[0, \underset{\|A\|}{2}]$, as outside this interval $f(t)>1$.

In the following we denote the lower and upper bounds in (21) by $X_{-}^{*}, X_{+}^{*}$ respectively. Observe that generically $\alpha^{*}<\lambda_{\min }(A)$ and $\beta^{*}>\lambda_{\max }(A)$ and hence $X_{-}^{*}=X\left(\alpha^{*}\right), X_{+}^{*}=X\left(\beta^{*}\right)$.

Due to the property that both matrices are positive definite, it follows immediately that $X_{-}^{*}$ is the solution that minimizes and $X_{+}^{*}$ is the solution that maximizes the determinant of $W(X)$ among all possible minimizers of the condition number.

\section{Special cases and examples}

In some special cases the solution to the problem of minimizing the condition number is much simpler than the one described in Theorem 1. It is clear that in the case that $A B^{\mathrm{H}} B=B^{\mathrm{H}} B A$ the solution can be reduced to finding the optimum out of the solution of $n$ quadratic equations, since $A$ and $B^{\mathrm{H}} B$ can be simultaneously diagonalized.

Even more special is the case that $A=I$. In this case problem (1) can be solved explicitly. Let $\sigma=\|B\|$, then the maximal eigenvalue of $t^{2} B^{\mathrm{H}} B+(I-t A)^{2}$ is $t^{2} \sigma^{2}+(1-t)^{2}$, so $f(t)=t^{2} \sigma^{2}+(1-t)^{2}$. A simple calculation shows that the minimum of $f(t)$ is $f^{*}=f\left(t^{*}\right)=\sigma^{2} /\left(\sigma^{2}+1\right)$ at $t^{*}=1 /\left(\sigma^{2}+1\right)$, and

$$
\frac{1+\sqrt{ } f^{*}}{1-\sqrt{ } f^{*}}=\left(\sqrt{ } 1+\sigma^{2}+\sigma\right)^{2} .
$$


Remark 1. It should be noted that by relaxing the requirement that $W(X)$ is positive definite, it is possible that the condition number can be made even smaller. Consider for example the matrix

$$
W(x)=\left[\begin{array}{ll}
1 & 1 \\
1 & x
\end{array}\right] .
$$

The condition number of $W(x)$ has two minima at $x_{1}=3$ and $x_{2}=-1$, where $\operatorname{cond}\left(W\left(x_{1}\right)\right)=3+\sqrt{ } 2$ and $\operatorname{cond}\left(W\left(x_{2}\right)\right)=1$. It is even $\operatorname{cond}(W(0)) \leq \operatorname{cond}\left(W\left(x_{1}\right)\right)$. The minimum among all positive definite completions is by (27) at $x_{1}=3$.

In order to further illustrate our results consider the following example:

Example 1. Let

$$
A=\left[\begin{array}{cccccc}
6 & & & & & \\
& 5 & & & & \\
& & 4 & & & \\
& & & 3 & & \\
& & & & 2 & \\
& & & & & 1
\end{array}\right], B^{\mathrm{T}}=\left[\begin{array}{ccc}
0.1 & 0.1 \\
0.2 & 0.0 \\
0.3 & 0.2 \\
0.4 & 0.0 \\
0.5 & 0.3 \\
0.6 & 0.0
\end{array}\right] .
$$

Since $\lambda_{\max }(A)=6$, the minimum of $f(t)$ is within the interval of $(0,0.3333)$. We have $t^{*}=0.2873$ and

$$
\alpha^{*}=0.9149, \quad \beta^{*}=6.0426 .
$$

The following Table 1 shows the distributions of the eigenvalues of $W\left(X^{*}\right)$ corresponding to $X_{-}^{*}$ and $X_{+}^{*}$.

Table 1. The eigenvalues of $W\left(X^{*}\right)$

\begin{tabular}{c|c|}
$X^{*}=X_{-}^{*}$ & $X^{*}=X_{+}^{*}$ \\
$\alpha^{*}$ & $\alpha^{*}$ \\
$\alpha^{*}$ & 1.9038 \\
2.0089 & 2.94562 \\
2.9453 & 3.9254 \\
3.9619 & 4.9541 \\
4.9555 & 5.5760 \\
5.7634 & $\beta^{*}$ \\
$\beta^{*}$ & $\beta^{*}$
\end{tabular}

It is not a special case that the smallest eigenvalues of $W\left(X_{-}^{*}\right)$ are multiple. In fact it is obvious that in general $W\left(X_{-}^{*}\right)$ has an eigenvalue $\alpha^{*}$ of multiplicity greater or equal to the dimension of $X$. The same holds for $W\left(X_{+}^{*}\right): W\left(X_{+}^{*}\right)$ has a largest eigenvalue $\beta^{*}$ of multiplicity greater than or equal to the dimension of $X$.

\section{References}

1. Arveson, W. (1975): Interpolation problems in nest algebras. J. Funct. Anal. 20, 208-233

2. Davis, C., Kahan, W.M., Weinberger, H.F. (1982): Norm-preserving dilations and their applications to optimal error bounds. SIAM J. Math. Anal. 19, 445-469

3. Elsner, L. He, C., Mehrmann, V.: Minimization of the norm, the norm of the inverse and the condition number of a matrix by completion. J. Numer. Lin. Algebra Applications (to appear) 
4. Elsner, L., He, C., Mehrmann, V.: Completion of a matrix so that the inverse has minimum norm. Application to the regularization of descriptor control problems. Linear Algebra for Control Theory, Paul Van Dooren, Bostwick Wyman, eds. The IMA Volumes in Mathematics and its Applications, Vol. 62, Springer-Verlag 1994

5. Gohberg, I., Rodman, L., Shalom, T., Woerdeman, H. (1993): Bounds for eigenvalues and singular values of matrix completions. Linear and Multilinear Algebra 33, 233-250

6. Golub, G.H., Van Loan, C.F. (1989) Matrix Computations, 2nd edn. The Johns Hopkins University Press, Baltimore, Maryland

7. Mehrmann, V. (1993): Divide and conquer methods for block tridiagonal linear systems. Parallel Computing 19, 257-279

8. Parrot, S. (1978): On the quotient norm and the Sz. Nagy-Foias lifting theorem. J. Funct. Anal. 30, 311-328

This article was processed by the author using the $\mathrm{LT}_{\mathrm{E}} \mathrm{X}$ style file pljourl from Springer-Verlag. 